\title{
Self-care of liver, kidney and bone marrow transplant patients with diabetes
}

Rafaella Roque Chagas ${ }^{1}$, Francisca Diana da Silva Negreiros*2, Tatiana Rebouças Moreira ${ }^{1}$, Samila Torquato Araújo ${ }^{1}$, Sherida Karanini Paz de Oliveira ${ }^{2}$, Maria de Jesus Nascimento de Aquino ${ }^{1}$, Açucena Leal de Araújo ${ }^{2}$, Thereza Maria Magalhães Moreira ${ }^{2}$, Lucilane Maria Sales da Silva ${ }^{2}$, Ana Célia Caetano de Sousa ${ }^{1}$, Yasmim Neri Pinheiro ${ }^{1}$, Gemiliana Sombra de Oliveira Carvalho ${ }^{1}$, Antônio Augusto Ferreira Carioca ${ }^{3}$

${ }^{1}$ Walter Cantídio University Hospital, Federal University of Ceará, Fortaleza, Brazil

${ }^{2}$ Public Health Graduate Program, Ceará State University, Fortaleza, Brazil

${ }^{3}$ Public Health Graduate Program, University of Fortaleza, Fortaleza, Brazil

Received: June 25, 2021

DOI: $10.5430 /$ jnep.v11n12p52
Accepted: July 30, 2021

Online Published: August 11, 2021

\begin{abstract}
Self-care is a set of actions that individuals take to maintain life, good health, and well-being. Regardless of the type of diabetes, individuals must perform self-care and comply with the treatment to prevent complications, achieve better disease management, and maintain their quality of life. This study aimed to examine self-care behaviors of liver, kidney, and bone marrow transplant patients with diabetes. The study has used a descriptive correlational design and was carried out in an endocrinology and diabetes center in Brazil. A total of 101 patients participated in the study. The Diabetes Self-care Activities Questionnaire was used, and the quantitative analysis was carried out using SPSS. The results show that the highest self-care levels occurred in the medication domain, while the lowest were found in the specific diet domain. Some important correlations were found: men were more likely to assess blood glucose, use combined oral/insulin therapy, take insulin, and take medications as prescribed than women; patients on combined oral/insulin therapy followed dietary recommendations more frequently than the others; and patients with altered serum urea and history of stroke had high levels of self-care. The results made it possible to know the compliance in performing self-care activities in transplanted patients with diabetes, supporting the development of interventions to motivate and improve self-care.
\end{abstract}

Key Words: Diabetes mellitus, Transplant recipients, Self care, Patient compliance, Health promotion

\section{INTRODUCTION}

Diabetes mellitus (DM) is a public health problem with high healthcare costs due to hospital admissions, complications, and decompensated conditions. The International Diabetes Federation (IDF) estimates annual global health expenditures for diabetes of US $\$ 760$ billion, and it is predicted that this value will reach US $\$ 845$ billion in 2045 , in the age group of 18 to 99 years. $^{[1]}$ The disease is classified according to its etiology, as follows: type 1 diabetes mellitus (DM1), type 2 diabetes mellitus (DM2), gestational diabetes mellitus (GDM), and other specific types, including post-transplant diabetes mellitus (PTDM). ${ }^{[2]}$

The number of transplantations is growing worldwide, but it is still below the level needed to reach all patients. PTDM

\footnotetext{
*Correspondence: Francisca Diana da Silva Negreiros; Email: negreiros.diana@gmail.com; Address: Clinical Care in Nursing and Health Program, Ceara State University - 1290 Pastor Samuel Munguba St., Fortaleza, Ceara, Brazil.
} 
has an unfavorable impact on the transplant graft survival and on the patient's survival. The use of immunosuppressive drugs is crucial for the success of transplants and is closely related to PTDM. ${ }^{[2]}$

Complications related to DM occur due to the long period of illness and poor glycemic control. After diagnosing diabetes, glycemic control is the main target of the treatment to prevent or delay acute and chronic complications, enabling greater quality of life. ${ }^{[3]}$ For this, health professionals must make patients aware of the importance of self-care, the practice of activities to maintain life, health, and well-being. ${ }^{[4]}$ Effective diabetes and transplantation management require self-care behaviors that are important to prevent complications and promote the quality of life. ${ }^{[5]}$

Studies show that self-care practices performed by patients with DM are related to improved glycemic control, ${ }^{[6]}$ decreased risk of complications, ${ }^{[7]}$ reduced use of healthcare resources, ${ }^{[8]}$ and improved quality of life. ${ }^{[9]}$ Therefore, healthcare professionals must assess compliance with self-care activities, to create individualized and adequate therapeutic plans. ${ }^{[10,11]}$

National ${ }^{[12,13]}$ and international ${ }^{[14,15]}$ studies have sought to understand the self-care behaviors of patients with diabetes and the relationships between clinical and socioeconomic variables and self-care. However, studies with transplanted patients with diabetes are scarce, and no studies have included an assessment of self-care behaviors in this population of patients.

It is important to assess self-care practices of transplanted patients with diabetes and to identify factors that influence the behaviors adopted by these patients. This diagnosis may support the development of effective interventions that positively impact the metabolic parameters of patients and, consequently, strengthen the quality of healthcare, with an emphasis on supported self-care. This study aimed to examine self-care behaviors of liver, kidney, and bone marrow transplant patients with diabetes.

\section{MATERIALS AND METHODS}

The STROBE guidelines were followed in reporting this study. The study was approved by the Ethics Committee of the Federal University of Ceará, Brazil (protocol No. 2.613.740), and all participants signed a written informed consent form.

\subsection{Study design and setting}

This study has used a descriptive correlational design to assess self-care behaviors of transplanted patients with diabetes mellitus. The research was conducted in an endocrinology and diabetes center in the city of Fortaleza, Brazil, from May to November 2018.

\subsection{Study population}

There were 136 transplanted patients with diabetes monitored at the endocrinology center (study population) at the beginning of the study. The center assists transplanted patients every Friday in the morning and afternoon. During the study, 12 appointments were previously scheduled for each period of the day, totaling 24 patients seen by the multidisciplinary team per day. As the researchers wanted to include the maximum number of subjects from the study location, no sample size calculation was undertaken. Inclusion criteria were transplanted patients with DM (diagnosed previously or after-transplantation), who were continuously monitored at the endocrinology center, able to understand, verbalize, answer questions, and with clinical conditions to participate. Patients who missed appointments during data collection were excluded. After applying the criteria, 136 patients were invited to participate. The main researcher carried out the recruitment consecutively (as patients arrived), but 35 refused. Thus, 101 patients participated in the study.

\subsection{Procedures}

Data were collected using two instruments: (1) a form with sociodemographic, clinical, and laboratory data, and (2) the Diabetes Self-Care Activities Questionnaire (translated and validated to the Portuguese language). The first instrument was developed by the researchers and included the following variables: age, gender, race, city of origin, marital status, education, religion, occupation, type and time of transplant, type of DM and time of diagnosis, clinical history, and associated complications.

The second instrument, the Diabetes Self-Care Activities Questionnaire (DSCAQ), translated and validated to the Portuguese language, was created based on the Summary of Diabetes Self-Care Activities Questionnaire, which accesses diabetic patients' self-care. The DSCAQ is a scale composed of six aspects of the diabetes regimen: general diet, specific diet, exercise, glucose monitoring, foot care, medication taking, and smoking. The assessment is standardized on weekdays, with scores indicating performance in self-care activities, on a scale from zero to seven (zero corresponds to the least desirable response and seven to the most favorable, except for specific diet, which has reverse scoring). ${ }^{[16]}$ Smoking assessment is coded separately and has not been addressed in our study. Although the DSCAQ does not provide a score specifically created to assess self-care, in this study, we considered an average DSCAQ $>4$ as indicative of the desirable performance of self-care activities, as it has 
been done in a previous study. ${ }^{[12]}$

The data collection was conducted with each patient after their medical consultations in a reserved room, and each encounter lasted 40 minutes on average. The research team carefully planned the data collection and participated in a training to improve the data collection consistency and reduce bias.

\subsection{Statistical analysis}

Data were entered and analyzed using the Statistical Package for Social Sciences (SPSS) software, version 22.0. MannWhitney U, Kruskal-Wallis, Student's $t$, and Fisher's Exact tests were used to analyze the data.

\section{Results}

Of 101 transplanted patients with DM, there was a predominance of males $(67 ; 66.3 \%)$, with age $<60$ years $(64 ; 63.4 \%)$, and brown skin $(57 ; 56.6 \%)$. Forty-seven $(46.6 \%)$ subjects had finished elementary school. Regarding marital status, most participants $(67 ; 66.3 \%)$ were married or partnered, $86(85.1 \%)$ had government benefits, and $70(69.3 \%)$ had a monthly family income of one to two minimum wages (see Table 1).

As for the time of diagnosis of DM, 54 (53.5\%) subjects had been diagnosed for less than ten years. The main types of diabetes found were DM2 $(50,49.5 \%)$ and PTDM (48, 47.5\%). Regarding the types of transplants, most subjects had undergone kidney transplantation $(50,49.5 \%)$. Systemic arterial hypertension $(52,51.5 \%)$ and dyslipidemia $(49,48.5 \%)$ were the most frequent comorbidities, and retinopathy $(23,22.8 \%)$ and diabetic kidney disease $(22,21.8 \%)$ were the most frequent complications (see Table 1).

The results on self-care behaviors demonstrated that the highest-level behavior in which the patients engaged was 'taking medication as recommended' (oral/insulin therapy) $(6.47 \pm 1.66)$ followed by 'not eating sweets' $(0.89 \pm 1.63$, Table 2).

Regarding self-care behaviors, men assessed blood glucose more frequently than women $(p=.009)$. It was also observed that men had a better engagement in the behavior of 'taking medications as recommended in the last week', with a statistically significant difference in the groups using combined oral/insulin therapy $(p=.008)$, insulin therapy $(p=.003)$, and oral therapy $(p=.046)$ (see Table 3$)$.
Table 1. Sociodemographic characterization, type and time of diagnosis of diabetes, type and time of transplantation, clinical history, and complications

\begin{tabular}{|c|c|c|c|}
\hline Variables & $\mathbf{n}$ & $\%$ & $95 \% \mathrm{CI}$ \\
\hline \multicolumn{4}{|l|}{ Gender } \\
\hline Male & 67 & 66.3 & $56.8-75$ \\
\hline Female & 34 & 33.7 & $25-43.2$ \\
\hline \multicolumn{4}{|l|}{ Age (in years) } \\
\hline$<60$ & 64 & 63.4 & $53.7-72.3$ \\
\hline$\geq 60$ & 37 & 36.6 & $27.7-46.3$ \\
\hline \multicolumn{4}{|l|}{ Skin color } \\
\hline Brown & 57 & 56.6 & $46.7-65.8$ \\
\hline Black & 14 & 13.9 & $8.2-21.6$ \\
\hline White & 28 & 27.7 & $19.7-37$ \\
\hline Yellow & 2 & 2.0 & $0.4-6.2$ \\
\hline \multicolumn{4}{|l|}{ Educational status } \\
\hline Elementary school & 47 & 46.6 & $37-56.2$ \\
\hline High school & 37 & 36.7 & $27.7-46.3$ \\
\hline Higher education & 17 & 16.9 & $10.5-25$ \\
\hline \multicolumn{4}{|l|}{ Marital status } \\
\hline Married/partnered & 67 & 66.3 & $56.8-75$ \\
\hline Single & 34 & 33.7 & $25-43.2$ \\
\hline \multicolumn{4}{|l|}{ Working status } \\
\hline Retired/Retired due to disease/Pensioner & 86 & 85.1 & $77.3-91.1$ \\
\hline Employed & 11 & 10.9 & $5.9-18.1$ \\
\hline Unemployed & 4 & 4.0 & $1.4-9.1$ \\
\hline \multicolumn{4}{|l|}{ Family income $^{\dagger}$} \\
\hline 1-2 minimum wages & 70 & 69.3 & $59.9-77.7$ \\
\hline$\geq 3$ minimum wages & 31 & 30.7 & $22.3-40.1$ \\
\hline \multicolumn{4}{|l|}{ Time of diagnosis of diabetes } \\
\hline$<10$ years & 54 & 53.5 & $43.8-63$ \\
\hline$\geq 10$ years & 47 & 46.5 & $37-56.2$ \\
\hline \multicolumn{4}{|l|}{ Type of diabetes } \\
\hline DM2 & 50 & 49.5 & $39.9-59.2$ \\
\hline PTDM & 48 & 47.5 & $38-57.2$ \\
\hline DM1 & 2 & 2.0 & $0.4-6.2$ \\
\hline MODY* & 1 & 1.0 & $0.1-4.5$ \\
\hline \multicolumn{4}{|l|}{ Type of transplant } \\
\hline Kidney & 50 & 49.5 & $39.9-59.2$ \\
\hline Liver & 49 & 48.5 & $38.9-58.2$ \\
\hline Bone marrow & 2 & 2.0 & $0.4-6.2$ \\
\hline \multicolumn{4}{|l|}{ Clinical history ${ }^{\S}$} \\
\hline Systemic hypertension & 52 & 51.5 & 41.8-61.1 \\
\hline Dyslipidemia & 49 & 48.5 & $38.9-58.2$ \\
\hline Cirrhosis & 34 & 33.7 & $25-43.2$ \\
\hline Thyroid diseases & 14 & 13.9 & $8.2-21.6$ \\
\hline Hepatitis & 12 & 11.9 & $5.6-18.2$ \\
\hline \multicolumn{4}{|l|}{ Complications $^{\S}$} \\
\hline Hypoglycemia & 29 & 28.7 & $16.3-41.1$ \\
\hline Diabetic retinopathy & 23 & 22.8 & $15.4-31.6$ \\
\hline Diabetic kidney disease & 22 & 21.8 & 14.6-30.6 \\
\hline Diabetic neuropathy & 21 & 20.8 & $13.8-29.5$ \\
\hline Peripheral vascular disease & 14 & 13.9 & $8.2-21.6$ \\
\hline Stroke & 8 & 7.9 & $3.8-14.4$ \\
\hline Diabetic foot & 7 & 6.9 & $3.2-13.1$ \\
\hline Hyperosmolar hyperglycemic state & 3 & 3.0 & $0.8-7.7$ \\
\hline Ischemic heart disease & 2 & 2.0 & $0.4-6.2$ \\
\hline Diabetic ketoacidosis & 1 & 1.0 & $0.1-4.5$ \\
\hline Amputation & 1 & 1.0 & $0.1-4.5$ \\
\hline
\end{tabular}


Table 2. Self-care behaviors measured using the diabetes self-care activities questionnaire

\begin{tabular}{lc}
\hline DSCAQ items & Total (SD) \\
\hline General diet & $5.80 \pm 2.50$ \\
1. Follow a healthy diet & $3.74 \pm 2.97$ \\
2. Follow dietary recommendations & \\
Specific diet & $1.82 \pm 2.73$ \\
3. Eating five or more portions of fruits and/or vegetables & $3.81 \pm 2.78$ \\
4. Eating red meats and/or whole milk derivates & $0.89 \pm 1.63$ \\
5. Eating sweets & \\
Exercise & $2.61 \pm 2.76$ \\
6. Exercise for at least 30 minutes & $1.83 \pm 2.61$ \\
7. Perform a specific exercise & \\
Blood glucose testing & $4.47 \pm 2.77$ \\
8. Assessing blood glucose & $3.00 \pm 2.93$ \\
9. Assessing blood glucose as frequently as recommended & \\
Foot care & $4.26 \pm 3.31$ \\
10. Checking feet & $3.42 \pm 3.42$ \\
11. Inspecting inside shoes before putting them on & $4.54 \pm 3.12$ \\
12. Drying between toes after washing feet & \\
Medication & $6.47 \pm 1.66$ \\
13. Taking medication as recommended & $6.14 \pm 1.86$ \\
14. Taking insulin as recommended & $6.31 \pm 1.95$ \\
15. Taking the indicated number of anti-diabetic pills &
\end{tabular}

Regarding 'taking medication as recommended' in the last week, a statistically significant difference was found for age groups, with the highest-level behaviors in people aged $<60$ years $(<60$ years: $6.63 \pm 1.41 ; \geq 60$ years: $5.75 \pm 2.58$; $p=.039)$. There was also a significant difference between people with elementary, high, and higher education concerning 'eating sweets' $(p=.006)$, and a significant difference regarding 'exercise for at least 30 minutes' between individuals with different educational levels $(p=.019)$. Two-by-two comparisons were made and showed that people with high school education ate more sweets in the last week $(1.49 \pm$ $1.95)$ than people with elementary education $(0.45 \pm 1.14)$ ( $p$ $=.004)$. The frequency of physical activity was higher in the high school group $(3.51 \pm 2.67)$ compared to the elementary school group $(1.85 \pm 2.56)(p=.004$, see Table 3$)$.

People diagnosed with diabetes for less than ten years had a better engagement in 'eating five or more portions of fruits or vegetables' $(2.35 \pm 2.98 ; p=.048)$. On the other hand, people with diabetes for more than ten years monitored their blood glucose $(5.47 \pm 2.44 ; p=.001)$ and assessed their blood in the recommended frequency $(3.64 \pm 3.02 ; p=.044)$ more often than those with diabetes for less than ten years (see Table 3).

The analysis of associations between DSCAQ scores and type of pharmacological treatment classified as (1) oral therapy, $=.008$ ).
(2) insulin therapy, and (3) combined oral/insulin therapy resulted a statistically significant difference in the following items: following a healthy diet ( $p=.043$ ), assessing blood glucose $(p=.001)$, assessing blood glucose as frequently as recommended $(p=.000)$, and taking insulin as recommended $(p=.031)$ (see Table 4). Two-by-two comparisons showed that the subjects using combined oral/insulin therapy had a better engagement in 'having a healthy diet' than those using oral therapy $(p=.043)$. In addition, people who were using insulin therapy assessed their blood glucose more frequently than those using oral therapy $(p=.001)$.

Associations between laboratory test variables and a desirable self-care behavior (DSCAQ > 4) were tested. The desirable self-care behavior was exhibited more often in people who had altered levels of urea $(p=.048)$ (see Table 5).

Associations between the presence/absence of complications (hypoglycemia, diabetic ketoacidosis, hyperosmolar hyperglycemic state, retinopathy, kidney disease, neuropathy, peripheral vascular disease, diabetic foot, amputation, ischemic heart disease, and stroke) and a desirable self-care behavior (DSCAQ > 4) were also tested. The only significant association found was between a history of stroke and DSCAQ $>4$ (persons with a history of stroke exhibited a desirable self-care behavior significantly more often than the others, $p$ $.008)$ 
Table 3. Analysis of self-care behaviors according to gender, age, education, and time of diagnosis

\begin{tabular}{|c|c|c|c|c|c|c|c|c|c|c|c|c|c|}
\hline \multirow{2}{*}{ DSCAQ items } & \multicolumn{3}{|c|}{ Gender } & \multicolumn{3}{|l|}{ Age } & \multicolumn{4}{|l|}{ Education } & \multicolumn{3}{|c|}{$\begin{array}{l}\text { Time of diagnosis } \\
\text { (years) }\end{array}$} \\
\hline & $\mathbf{F}$ & $\mathbf{M}$ & $p$ & $\begin{array}{l}< \\
60\end{array}$ & $\begin{array}{l}\geq \\
60\end{array}$ & $p$ & Elementary & High & Higher & $p$ & $\begin{array}{l}< \\
10\end{array}$ & $\begin{array}{l}\geq \\
10\end{array}$ & $p$ \\
\hline \multicolumn{14}{|l|}{ General diet } \\
\hline $\begin{array}{l}\text { 1. Follow a } \\
\text { healthy diet }\end{array}$ & $\begin{array}{l}4.94 \\
\pm \\
2.35\end{array}$ & $\begin{array}{l}5.15 \\
\pm \\
2.29\end{array}$ & .472 & $\begin{array}{l}4.89 \\
\pm \\
2.59\end{array}$ & $\begin{array}{l}5.41 \\
\pm \\
2.33\end{array}$ & .292 & $5.34 \pm 2.32$ & $\begin{array}{l}4.51 \pm \\
2.69\end{array}$ & $\begin{array}{l}5.59 \pm \\
2.48\end{array}$ & .171 & $\begin{array}{l}5.24 \\
\pm \\
2.28\end{array}$ & $\begin{array}{l}4.89 \\
\pm \\
2.74\end{array}$ & .820 \\
\hline $\begin{array}{l}\text { 2. Follow dietary } \\
\text { recommendations }\end{array}$ & $\begin{array}{l}3.59 \\
\pm \\
2.90\end{array}$ & $\begin{array}{l}3.82 \\
\pm \\
3.02\end{array}$ & .661 & $\begin{array}{l}3.45 \\
\pm \\
2.97\end{array}$ & $\begin{array}{l}4.24 \\
\pm \\
2.94\end{array}$ & .162 & $3.94 \pm 2.94$ & $\begin{array}{l}3.22 \pm \\
3.02\end{array}$ & $\begin{array}{l}4.35 \pm \\
2.91\end{array}$ & .341 & $\begin{array}{l}4.13 \\
\pm \\
2.84\end{array}$ & $\begin{array}{l}3.30 \\
\pm \\
3.09\end{array}$ & .202 \\
\hline \multicolumn{14}{|l|}{ Specific diet } \\
\hline $\begin{array}{l}\text { 3. Eating five or } \\
\text { more portions of } \\
\text { fruits and/or } \\
\text { vegetables }\end{array}$ & $\begin{array}{l}1.41 \\
\pm \\
2.46\end{array}$ & $\begin{array}{l}2.03 \\
\pm \\
2.85\end{array}$ & .39 & $\begin{array}{l}1.92 \\
\pm \\
2.76\end{array}$ & $\begin{array}{l}1.65 \\
\pm \\
2.70\end{array}$ & .558 & $2.04 \pm 2.86$ & $\begin{array}{l}1.32 \pm \\
2.29\end{array}$ & $\begin{array}{l}2.29 \pm \\
3.22\end{array}$ & .411 & $\begin{array}{l}2.35 \\
\pm \\
2.98\end{array}$ & $\begin{array}{l}1.21 \\
\pm \\
2.29\end{array}$ & $.048^{*}$ \\
\hline $\begin{array}{l}\text { 4. Eating red } \\
\text { meats and/or } \\
\text { whole milk } \\
\text { derivates }\end{array}$ & $\begin{array}{l}3.91 \\
\pm \\
2.64\end{array}$ & $\begin{array}{l}3.76 \\
\pm \\
2.86\end{array}$ & .551 & $\begin{array}{l}3.69 \\
\pm \\
2.71\end{array}$ & $\begin{array}{l}4.03 \\
\pm \\
2.92\end{array}$ & .703 & $3.94 \pm 2.93$ & $\begin{array}{l}4.14 \pm \\
2.57\end{array}$ & $\begin{array}{l}2.76 \pm \\
2.68\end{array}$ & .195 & $\begin{array}{l}3.52 \\
\pm \\
2.79\end{array}$ & $\begin{array}{l}4.15 \\
\pm \\
2.75\end{array}$ & .155 \\
\hline 5. Eating sweets & $\begin{array}{l}1.18 \\
\pm \\
1.75\end{array}$ & $\begin{array}{l}0.75 \\
\pm \\
1.56\end{array}$ & .126 & $\begin{array}{l}1.03 \\
\pm \\
1.73\end{array}$ & $\begin{array}{l}0.65 \\
\pm \\
1.44\end{array}$ & .100 & $0.45 \pm 1.14$ & $\begin{array}{l}1.49 \pm \\
1.95\end{array}$ & $\begin{array}{l}0.82 \pm \\
1.70\end{array}$ & $.006^{* *}$ & $\begin{array}{l}0.81 \\
\pm \\
1.49\end{array}$ & $\begin{array}{l}0.98 \\
\pm \\
1.79\end{array}$ & .929 \\
\hline \multicolumn{14}{|l|}{ Exercise } \\
\hline $\begin{array}{l}\text { 6. Exercise for at } \\
\text { least } 30 \text { minutes }\end{array}$ & $\begin{array}{l}2.5 \\
\pm \\
2.67\end{array}$ & $\begin{array}{l}2.67 \\
\pm \\
2.82\end{array}$ & .916 & $\begin{array}{l}2.86 \\
\pm \\
2.73\end{array}$ & $\begin{array}{l}2.19 \\
\pm \\
2.79\end{array}$ & .223 & $1.85 \pm 2.56$ & $\begin{array}{l}3.51 \pm \\
2.67\end{array}$ & $\begin{array}{l}2.76 \pm \\
2.68\end{array}$ & $.019^{*}$ & $\begin{array}{l}2.15 \\
\pm \\
2.63\end{array}$ & $\begin{array}{l}1.47 \\
\pm \\
2.56\end{array}$ & .73 \\
\hline $\begin{array}{l}\text { 7. Perform a } \\
\text { specific exercise }\end{array}$ & $\begin{array}{l}2.18 \\
\pm \\
2.69\end{array}$ & $\begin{array}{l}1.66 \\
\pm \\
2.57\end{array}$ & .185 & $\begin{array}{l}1.83 \\
\pm \\
2.57\end{array}$ & $\begin{array}{l}1.84 \\
\pm \\
2.71\end{array}$ & .971 & $1.47 \pm 2.36$ & $\begin{array}{l}2.41 \pm \\
2.82\end{array}$ & $\begin{array}{l}1.59 \pm \\
2.72\end{array}$ & .337 & $\begin{array}{l}2.15 \\
\pm \\
2.63\end{array}$ & $\begin{array}{l}1.47 \\
\pm \\
2.56\end{array}$ & .135 \\
\hline \multicolumn{14}{|l|}{$\begin{array}{l}\text { Blood glucose } \\
\text { testing }\end{array}$} \\
\hline $\begin{array}{l}\text { 8. Assessing blood } \\
\text { glucose }\end{array}$ & $\begin{array}{l}3.56 \\
\pm \\
2.80\end{array}$ & $\begin{array}{l}4.93 \\
\pm \\
2.65\end{array}$ & $.009^{* *}$ & $\begin{array}{l}4.19 \\
\pm \\
2.71\end{array}$ & $\begin{array}{l}4.95 \\
\pm \\
2.83\end{array}$ & .192 & $4.85 \pm 2.73$ & $\begin{array}{l}3.62 \pm \\
2.78\end{array}$ & $\begin{array}{l}5.24 \pm \\
2.49\end{array}$ & .81 & $\begin{array}{l}3.59 \\
\pm \\
2.76\end{array}$ & $\begin{array}{l}5.47 \\
\pm \\
2.44\end{array}$ & $.001^{* *}$ \\
\hline $\begin{array}{l}\text { 9. Assessing blood } \\
\text { glucose as } \\
\text { frequently as } \\
\text { recommended }\end{array}$ & $\begin{array}{l}2.35 \\
\pm \\
2.74\end{array}$ & $\begin{array}{l}3.33 \\
\pm \\
2.99\end{array}$ & .112 & $\begin{array}{l}2.78 \\
\pm \\
2.81\end{array}$ & $\begin{array}{l}3.38 \\
\pm \\
3.13\end{array}$ & .420 & $2.96 \pm 2.93$ & $\begin{array}{l}2.62 \pm \\
2.93\end{array}$ & $\begin{array}{l}3.94 \pm \\
2.90\end{array}$ & .219 & $\begin{array}{l}2.44 \\
\pm \\
2.77\end{array}$ & $\begin{array}{l}3.64 \\
\pm \\
3.02\end{array}$ & $.044^{*}$ \\
\hline \multicolumn{14}{|l|}{ Foot care } \\
\hline 10. Checking feet & $\begin{array}{l}4.29 \\
\pm \\
3.32\end{array}$ & $\begin{array}{l}4.24 \\
\pm \\
3.34\end{array}$ & .85 & $\begin{array}{l}4.36 \\
\pm \\
3.27\end{array}$ & $\begin{array}{l}4.08 \\
\pm \\
3.42\end{array}$ & .629 & $4.09 \pm 3.34$ & $\begin{array}{l}4.70 \pm \\
3.21\end{array}$ & $\begin{array}{l}3.76 \pm \\
3.54\end{array}$ & .562 & $\begin{array}{l}4.22 \\
\pm \\
3.32\end{array}$ & $\begin{array}{l}4.30 \\
\pm \\
3.34\end{array}$ & .870 \\
\hline $\begin{array}{l}\text { 11. Inspecting } \\
\text { inside shoes before } \\
\text { putting them on }\end{array}$ & $\begin{array}{l}2.76 \\
\pm \\
3.28\end{array}$ & $\begin{array}{l}3.75 \\
\pm \\
3.47\end{array}$ & .217 & $\begin{array}{l}2.95 \\
\pm \\
3.35\end{array}$ & $\begin{array}{l}4.22 \\
\pm \\
3.43\end{array}$ & .079 & $3.77 \pm 3.50$ & $\begin{array}{l}2.76 \pm \\
3.28\end{array}$ & $\begin{array}{l}3.88 \pm \\
3.48\end{array}$ & .375 & $\begin{array}{l}3.56 \\
\pm \\
3.50\end{array}$ & $\begin{array}{l}3.26 \\
\pm \\
3.35\end{array}$ & .751 \\
\hline $\begin{array}{l}\text { 12. Drying } \\
\text { between toes after } \\
\text { washing feet }\end{array}$ & $\begin{array}{l}4.85 \\
\pm \\
3.01\end{array}$ & $\begin{array}{l}4.39 \\
\pm \\
3.19\end{array}$ & .482 & $\begin{array}{l}4.31 \\
\pm \\
3.21\end{array}$ & $\begin{array}{l}4.95 \\
\pm \\
2.96\end{array}$ & .364 & $4.26 \pm 3.12$ & $\begin{array}{l}4.62 \pm \\
3.22\end{array}$ & $\begin{array}{l}5.18 \pm \\
2.96\end{array}$ & 0.393 & $\begin{array}{l}3.56 \\
\pm \\
3.50\end{array}$ & $\begin{array}{l}3.26 \\
\pm \\
3.35\end{array}$ & .207 \\
\hline \multicolumn{14}{|l|}{ Medication } \\
\hline $\begin{array}{l}\text { 13. Taking } \\
\text { medication as } \\
\text { recommended }\end{array}$ & $\begin{array}{l}5.85 \\
\pm \\
2.39\end{array}$ & $\begin{array}{l}6.78 \\
\pm \\
1.04\end{array}$ & $.008^{* *}$ & $\begin{array}{l}6.62 \\
\pm \\
1.41\end{array}$ & $\begin{array}{l}6.22 \\
\pm \\
2.02\end{array}$ & .114 & $6.47 \pm 1.65$ & $\begin{array}{l}6.27 \pm \\
1.98\end{array}$ & $\begin{array}{l}6.94 \pm \\
0.25\end{array}$ & .528 & $\begin{array}{l}6.28 \\
\pm \\
1.96\end{array}$ & $\begin{array}{l}6.68 \\
\pm \\
1.24\end{array}$ & .295 \\
\hline $\begin{array}{l}\text { 14. Taking insulin } \\
\text { as recommended }\end{array}$ & $\begin{array}{l}5.44 \\
\pm \\
2.19\end{array}$ & $\begin{array}{l}6.43 \\
\pm \\
1.66\end{array}$ & $.003^{* *}$ & $\begin{array}{l}5.92 \\
\pm \\
2.17\end{array}$ & $\begin{array}{l}6.55 \\
\pm \\
1.05\end{array}$ & .474 & $6.46 \pm 1.24$ & $\begin{array}{l}6.05 \pm \\
1.86\end{array}$ & $\begin{array}{l}5.44 \pm \\
3.09\end{array}$ & .595 & $\begin{array}{l}6.00 \\
\pm \\
2.16\end{array}$ & $\begin{array}{l}6.24 \\
\pm \\
1.67\end{array}$ & .757 \\
\hline $\begin{array}{l}\text { 15. Taking the } \\
\text { indicated number } \\
\text { of anti-diabetic } \\
\text { pills }\end{array}$ & $\begin{array}{l}5.87 \\
\pm \\
2.38\end{array}$ & $\begin{array}{l}6.55 \\
\pm \\
1.63\end{array}$ & $.046^{*}$ & $\begin{array}{l}6.63 \\
\pm \\
1.41\end{array}$ & $\begin{array}{l}5.75 \\
\pm \\
2.58\end{array}$ & $.039^{*}$ & $6.22 \pm 2.13$ & $\begin{array}{l}6.10 \pm \\
2.17\end{array}$ & $\begin{array}{l}6.94 \pm \\
0.25\end{array}$ & .456 & $\begin{array}{l}6.38 \\
\pm \\
1.75\end{array}$ & $\begin{array}{l}6.19 \\
\pm \\
2.23\end{array}$ & .923 \\
\hline
\end{tabular}

*Correlation is significant at the .05 level. **Correlation is significant at the .01 level. 
Table 4. Analysis of associations between self-care behaviors and pharmacological treatment types

\begin{tabular}{|c|c|c|c|c|}
\hline \multirow[b]{2}{*}{ DSCAQ items } & \multicolumn{3}{|c|}{ Pharmacological treatment types } & \multirow[b]{2}{*}{$p$} \\
\hline & $\begin{array}{l}\text { Oral therapy } \\
(n=46)\end{array}$ & $\begin{array}{l}\text { Insulin therapy } \\
(n=20)\end{array}$ & $\begin{array}{l}\text { Oral/insulin } \\
\text { therapy } \\
(n=35)\end{array}$ & \\
\hline \multicolumn{5}{|l|}{ General diet } \\
\hline 1. Follow a healthy diet & $5.04 \pm 2.33(5.50)$ & $4.30 \pm 3.16(7.00)$ & $5.57 \pm 2.24(7.00)$ & .323 \\
\hline 2. Follow dietary recommendations & $3.30 \pm 2.86(4.00)$ & $3.05 \pm 3.20(2.50)$ & $4.71 \pm 2.80(7.00)$ & $.043^{*}$ \\
\hline \multicolumn{5}{|l|}{ Specific diet } \\
\hline 3. Eating five or more portions of fruits and/or vegetables & $1.74 \pm 2.78(0.00)$ & $1.70 \pm 2.68(0.00)$ & $2.00 \pm 2.77(0.00)$ & .089 \\
\hline 4. Eating red meats and/or whole milk derivates & $3.54 \pm 2.75(3.00)$ & $3.95 \pm 3.10(4.00)$ & $4.09 \pm 2.66(3.00)$ & .673 \\
\hline 5. Eating sweets & $0.80 \pm 1.64(0.00)$ & $0.85 \pm 1.63(0.00)$ & $1.03 \pm 1.65(0.00)$ & .532 \\
\hline \multicolumn{5}{|l|}{ Exercise } \\
\hline 6. Exercise for at least 30 minutes & $2.84 \pm 2.76(3.00)$ & $2.15 \pm 3.13(0.00)$ & $2.57 \pm 2.56(3.00)$ & .394 \\
\hline 7. Perform a specific exercise & $2.11 \pm 2.68(0.00)$ & $1.50 \pm 2.74(0.00)$ & $1.66 \pm 2.47(0.00)$ & .454 \\
\hline \multicolumn{5}{|l|}{ Blood glucose testing } \\
\hline 8. Assessing blood glucose & $3.37 \pm 3.01(2.50)$ & $5.60 \pm 2.30(7.00)$ & $5.26 \pm 2.13(7.00)$ & $.001^{* *}$ \\
\hline 9. Assessing blood glucose as frequently as recommended & $1.70 \pm 2.67(0.00)$ & $3.90 \pm 2.67(4.00)$ & $4.20 \pm 2.76(4.00)$ & $.000^{* *}$ \\
\hline \multicolumn{5}{|l|}{ Foot care } \\
\hline 10. Checking feet & $3.65 \pm 3.43(5.00)$ & $5.25 \pm 3.11(7.00)$ & $4.49 \pm 3.18(7.00)$ & .158 \\
\hline 11. Inspecting inside shoes before putting them on & $3.30 \pm 3.46(1.00)$ & $3.65 \pm 3.50(5.00)$ & $3.43 \pm 3.41(3.00)$ & .932 \\
\hline 12. Drying between toes after washing feet & $4.35 \pm 3.21(7.00)$ & $4.65 \pm 3.12(7.00)$ & $4.74 \pm 3.08(7.00)$ & .832 \\
\hline \multicolumn{5}{|l|}{ Medication } \\
\hline 13. Taking medication as recommended & $6.27 \pm 2.04(7.00)$ & $6.45 \pm 1.76(7.00)$ & $6.74 \pm 0.89(7.00)$ & .587 \\
\hline 14. Taking insulin as recommended & $0.00 \pm-(0.00)$ & $6.45 \pm 1.76(7.00)$ & $6.14 \pm 1.65(7.00)$ & $.031^{*}$ \\
\hline 15. Taking the indicated number of anti-diabetic pills & $6.43 \pm 1.78(7.00)$ & $5.25 \pm 3.24(7.00)$ & $6.38 \pm 1.78(7.00)$ & .602 \\
\hline
\end{tabular}

*Correlation is significant at the .05 level. ${ }^{* *}$ Correlation is significant at the .01 level.

Table 5. Associations between laboratory test variables and desirable DSCAQ score

\begin{tabular}{|c|c|c|c|}
\hline \multirow{2}{*}{$\begin{array}{ll}\text { Laboratory } & \text { test } \\
\text { variables } & \end{array}$} & \multicolumn{2}{|l|}{ DSCAQ } & \multirow[t]{2}{*}{$\bar{p}$} \\
\hline & Score $\leq 4$ & Score $>4$ & \\
\hline HbA1C & $6.6(1.4)$ & $6.8(1.5)$ & .547 \\
\hline Fasting blood glucose & $163(53)$ & $167(63)$ & .769 \\
\hline Total cholesterol & $172(35)$ & $179(48)$ & .509 \\
\hline HDL-C & $44(17)$ & $46(17)$ & .507 \\
\hline LDL-C & $94(28)$ & $92(33)$ & .851 \\
\hline Triglycerides & $173(97)$ & 189 (123) & .556 \\
\hline TGO & $25(11)$ & $25(10)$ & .867 \\
\hline TGP & $30(21)$ & $35(28)$ & .329 \\
\hline Urea & $45.2(27.8)$ & $57.7(23.1)$ & .048 \\
\hline Creatinine & $1.3(0.5)$ & $1.4(0.7)$ & .396 \\
\hline
\end{tabular}

\section{Discussion}

The present study was conducted with liver, kidney, and bone marrow transplant patients with diabetes, including both people with diabetes before transplantation and patients who were affected by diabetes after transplantation. Such variations may imply heterogeneity in self-care patterns be- tween the two groups, since patients affected by diabetes after transplantation can develop better self-care than those with previous diabetes due to the complexity that transplantation imposes. Patients who acquire diabetes after transplantation may be more motivated to perform self-care/disease management activities, as they have already had a life-threatening clinical condition.

On the other hand, patients with diabetes before transplantation may have had insufficient compliance with self-care behaviors throughout their lives, and the transplant may have been a consequence of failure to manage diabetes. Such aspects must be considered. However, they were not analyzed in detail in this study, which represents a methodological limitation.

The use of validated instruments contributes to establishing a common language among health professionals and related areas. It consists of a relevant tool for evaluating responses to therapies, comparing data, understanding/studying problems, and enabling effective decision-making. The choice of the DSCAQ was made for this study because it allows investigat- 
ing the self-care level of transplanted patients with diabetes, making it possible to raise insights about the effectiveness of health interventions.

Most participants were male, corroborating the results of a study that measured the frequency of metabolic diseases in patients with liver transplantation and DM, of which $76.1 \%$ were men. ${ }^{[17]}$ Besides, most participants were young adults, different from the predominant age group from another study ( $\geq 60$ years old patients). ${ }^{[18]}$ We also observed that most patients $(36.7 \%)$ had finished high school, which is lower than the educational level found in a study conducted in Malaysia, in which $47.9 \%$ of respondents had finished high school. ${ }^{[19]}$ Similar to our findings, a study that described the quality of foot care in patients with diabetes pointed out that most participants were married (68.89\%). ${ }^{[20]}$

Regarding the clinical characteristics, it was found that most subjects had less than ten years of diagnosis of diabetes, similar to Jannoo \& Khan's ${ }^{[18]}$ findings that show an average of 9.97 years of diagnosis. Most participants in our study had DM2 and DMPT, and only two subjects had DM1. The low frequency of patients with DM1 may be related to the fact that the study was carried out in a specialist center for metabolic diseases that provides care primarily, but not exclusively, to adult patients, and type 1 diabetes usually occurs in children and adolescents. A survey carried out in the Slovak Republic with 133 people to identify risk factors for DM in patients after 12 months of transplantation also found a high rate of DMPT $(52 ; 38.3 \%)$. It was concluded that participants with undiagnosed DM2 were among those with DMPT. The authors assume that this is possibly the reason for the high incidence of DMPT. ${ }^{[21]}$

The most prevalent type of transplant in this study was the kidney transplant. This finding corroborates Dedinská et al., ${ }^{[21]}$ results that indicate an increase in the incidence of DM post-kidney transplantation (15\%-30\%), associated with a high prevalence of overweight, obesity, and end-stage renal disease.

Regarding the subjects' clinical history, a study on quality of life carried out in Italy with 302 diabetic patients found that the most representative comorbidities were systemic hypertension $(73.5 \%)$ and dyslipidemia $(57.3 \%),{ }^{[11]}$ corroborating with our findings.

Constant hyperglycemia causes an increased risk of cardiovascular diseases through various mechanisms (insulin resistance, inflammation, endothelial dysfunction, and toxic effects of glucose in the microvessels). ${ }^{[1]}$ In addition, hyperglycemia is related to underlying metabolic risks, such as hypertension, dyslipidemia, and central obesity.
Concerning the general diet, a self-care behavior assessment study conducted in Malaysia ${ }^{[20]}$ found that individuals with DM2 follow a healthy diet for 5.05 days per week ( \pm 1.97$)$, which is below the frequency found in our study. On the other hand, the same research found that the participants followed the dietary recommendation in an average of 5.02 days over the past month $( \pm 1.92)$, which is higher than the frequency found in our study.

There is little research on gender differences related to selfcare behaviors in people with diabetes. A survey conducted in Italy ${ }^{[22]}$ to describe self-care differences related to gender found that women exhibit self-care behaviors equal to or greater than men, in contrast to our findings. Another investigation ${ }^{[23]}$ found that active male workers exhibited inadequate self-care behaviors more often than women, and low income was associated with inadequate self-care in women.

In the present study, patients followed dietary recommendations in a lower frequency than that of a study carried out in Ghana, which found a higher frequency of eating five or more portions of fruits or vegetables $(3.25 \pm 2.11)$. The authors also observed that less than $15 \%$ of the participants consumed fruits and vegetables daily. ${ }^{[24]}$

The weekly frequency of eating sweets in this study was lower than the frequency found by Franz et al., ${ }^{[25]}$ in which people with diabetes moderately consumed carbohydrates (44\%-46\% of total calories). It is difficult to sustain a diet with reduced carbohydrates, and an individualized diet plan is essential for the patients' acceptance. In developed countries, individuals with diabetes are encouraged to decrease their consumption of refined carbohydrates and added sugars and regularly eat vegetables, fruits, dairy products (milk and yogurt), and whole grains. ${ }^{[3]}$

Regarding the non-pharmacological treatment of DM, a research carried out in a primary care center in Malaysia, with people with DM2, found low self-care scores related to the behavior 'perform a specific exercise' $(2.09 \pm 2.33),{ }^{[19]}$ similar to our study.

Concerning blood glucose testing, a study carried out in Ghana found an average weekly frequency of blood glucose testing below what was found in our study. However, it was observed that men $(2.36 \pm 1.02)$ had a higher frequency of this behavior than women $(2.07 \pm 0.40 ; p=.007),{ }^{[24]}$ which is similar to our results.

Blood glucose self-monitoring is a simple method that allows personalized assistance, contributes to the decision-making process, and motivates individual lifestyle changes. The lack of financial resources and the inconvenience involved in the procedure may be related to the lack of compliance with 
home blood glucose monitoring. ${ }^{[26]}$

Regarding foot care, a study carried out in Indonesia to describe foot self-care found an unsatisfactory level of this behavior, ${ }^{[27]}$ corroborating our study findings on the item 'inspecting inside shoes before putting them on'.

Foot care actions allow early identification of changes and prevent complications, such as amputation. Lack of knowledge on how to perform foot care, poor clinician-patient communication, inconvenience, and lack of financial resources are factors that impair this self-care behavior. ${ }^{[25]}$

A study evaluated self-care and glycemic control in a hospital in Delhi ${ }^{[28]}$ and found that $82.4 \%$ of the patients took medications as recommended, similar to our findings. The same research shows that $25.6 \%$ of the sample reported forgetfulness among the reasons for not using the medication as prescribed, while $20 \%$ self-modified the therapy due to changes in their symptoms and sensation of well-being.

The present investigation found better self-care in transplanted patients with complications related to DM, such as stroke, and increased urea levels suggesting renal dysfunction. These results suggest that the proper compliance with self-care behaviors by people with diabetes is often late, with improvements occurring after the onset of complications. Thus, the work of health professionals to promote awareness of these patients about the importance of self-care through diabetological education must be reinforced. Such findings corroborate the results from another study conducted in Recife, Brazil, which showed that comorbidities and complications related to DM were a protective factor for negative attitudes towards the disease. ${ }^{[29]}$ These results reinforce that the satisfactory acquisition of self-care behaviors is often late, increasing after the appearance of complications. Thus, healthcare professionals must acknowledge the importance of raising awareness in this population using educational interventions.
The main limitation found in this study was the fact that the self-care activities were assessed through self-reporting, which is a less reliable method for identifying behaviors than testing people's self-management abilities. However, such a method proved to be practical and objective. Other potential limitation stem from the fact that the study was conducted in a single health center, thus restricting the generalizability of the results. Finally, the study was carried out with patients having pre- and post-transplantation diabetes, which may have implied different self-care patterns. However, such stratification was not addressed in the present study.

\section{Conclusions}

The highest-level self-care behavior shown by transplanted persons with DM was the use of combined oral/insulin therapy followed by not eating sweets. Some important correlations were found: men were more likely to assess blood glucose, use combined oral/insulin therapy, take insulin, and take medications as recommended than women; patients on combined oral/insulin therapy followed dietary recommendations more frequently than the others; and patients with altered serum urea and history of stroke had higher levels of self-care.

The knowledge about the frequency of self-care behaviors in transplanted people with diabetes is relevant for developing intervention programs aimed at this population. The support from a multi-professional team favors patients' motivation and self-care, including achieving adequate glycemic control and reducing diabetes-related complications, thus providing a better quality of life. In addition, it is necessary to assess the evolution of self-care behaviors through longitudinal studies to confirm the effects of health education actions.

\section{Conflicts of InTEREST Disclosure}

The authors declare that there is no conflict of interest.

\section{REFERENCES}

[1] International Diabetes Federation (IDF). IDF Diabetes Atlas. 9th ed. IDF; 2019

[2] Brazilian Diabetes Society (BDS). Brazilian Diabetes Society Guidelines (2019-2020). Clannad; 2020.

[3] American Diabetes Association (ADA). Standards of medical care in diabetes - 2020. Diabetes Care. 2020; 43: S1LP-S2. https: //doi.org/10.2337/dc20-Sint

[4] Ciske KL, Orem DE. Nursing: Concepts of practice. Am J Nurs. 1980; 80(10). https : //doi.org/10.2307/3462482

[5] Lee YJ, Shin SJ, Wang RH, et al. Pathways of empowerment perceptions, health literacy, self-efficacy, and self-care behaviors to glycemic control in patients with type 2 diabetes mellitus. Patient Educ Couns. 2016; 99(2): 287-94. https://doi.org/10.1016/ j.pec. 2015.08.021

[6] Houle J, Beaulieu MD, Chiasson JL, et al. Glycaemic control and self-management behaviours in Type 2 diabetes: Results from a 1year longitudinal cohort study. Diabet Med. 2015; 32(9): 1247-54. https ://doi.org/10.1111/dme.12686

[7] Ausili D, Rossi E, Rebora P, et al. Sociodemographic and clinical determinants of self-care in adults with type 2 diabetes: a multicentre observational study. Acta diabetol. 2018; 55: 691-702. https://doi .org/10.1007/s00592-018-1135-x

[8] Adepoju OE, Bolin JN, Phillips CD, et al. Effects of diabetes self- 
management programs on time-to-hospitalization among patients with type 2 diabetes: A survival analysis model. Patient Educ Couns. 2014; 95(1): 111-7. https://doi.org/10.1016/j.pec.2014.0 1.001

[9] Ildarabadi EH, Tabei MG, Khosh AM. Effects of face-to-face and online training on self-care of middle-aged and elderly people with type 2 diabetes: A comparative study. Open Access Maced J Med Sci. 2019; 7(7): 1214-9. https://doi.org/10.3889/oamjms.2 019.275

[10] Sousa VD, Hartman SW, Miller EH, et al. New measures of diabetes self-care agency, diabetes self-efficacy, and diabetes self-management for insulin-treated individuals with type 2 diabetes. J Clin Nurs. 2009; 18(9): 1305-12. https://doi.org/10.1111/j.1365-2702.20 08.02729.x

[11] Ausili D, Bulgheroni M, Ballatore P, et al. Self-care, quality of life and clinical outcomes of Type 2 Diabetes patients: An observational cross-sectional study. Acta diabetol. 2017; 54: 1001-8. https://doi.org/10.1007/s00592-017-1035-5

[12] Eid LP, Leopoldino SAD, Oller GASAO, et al. Factors related to self-care activities of patients with Type 2 Diabetes Mellitus. Esc Anna Nery Rev Enferm. 2018; 22: e20180046. https://doi.org/ 10.1590/2177-9465-ean-2018-0046

[13] Marques MB, Coutinho JFV, Martins MC, et al. Educational intervention to promote self-care in older adults with diabetes mellitus. Rev Esc Enferm USP. 2019; 53: e03517. https : //doi.org/10.1 590/s1980-220x2018026703517

[14] Kassa RN, Ibrahim IY, Hailemariam HA, et al. Self-care practice and its predictors among adults with diabetes mellitus on follow up at public hospitals of Arsi zone, southeast Ethiopia. BMC Res Notes. 2021; 14(1): 102. https://doi.org/10.1186/s13104-021-05511-0

[15] Al-Qahtani AM. Frequency and factors associated with inadequate self-care behaviors in patients with type 2 diabetes mellitus in $\mathrm{Na}$ jran, Saudi Arabia based on diabetes self-management questionnaire. Saudi Med J. 2020; 41(9): 955-964. http://dx.doi . org/10.15 $537 / \mathrm{smj} .2020 \cdot 9 \cdot 2533$

[16] Michels MJ, Coral MHC, Sakae TM, et al. Questionnaire of diabetes self-care activities: Translation, cross-cultural adaptation and evaluation of psychometric properties. Arq Bras Endocrinol Metabol. 2010; 54(7). https://doi .org/10.1590/s0004-27302010000 700009

[17] Moura Neto A, Bovi TG, Righetto CM, et al. Clinical profile of patients with diabetes mellitus and liver transplantation: Results after a multidisciplinary team intervention. Transplant Proc. 2018; 50(3): 784-7. https://doi.org/10.1016/j.transproceed.2 018.02 .042

[18] Jannoo Z, Khan NM. Medication Adherence and Diabetes Self-Care Activities among patients with Type 2 Diabetes Mellitus. Value Health Reg Issues. 2019; 18: 30-5. https://doi.org/10.101 $6 / j \cdot$ vhri.2018.06.003
[19] Tharek Z, Ramli AS, Whitford DL, et al. Relationship between selfefficacy, self-care behaviour and glycaemic control among patients with type 2 diabetes mellitus in the Malaysian primary care setting. BMC Fam Pract. 2018; 19(39). https://doi.org/10.1186/s1 2875-018-0725-6

[20] Swiątoniowska N, Chabowski M, Jankowska-Polańska B. Quality of foot care among patients with Diabetes: A study using a Polish version of the Diabetes Foot Disease and Foot Care Questionnaire. J Foot Ankle Res. 2020; 59(2): 231-8. https://doi .org/10.105 $3 / j \cdot j$ fas.2019.07.020

[21] Dedinská L, Baltesová T, Beňa L, et al. Incidence of Diabetes Mellitus after kidney transplantation in Slovakia: Multicentric, prospective analysis. Transplant Proc. 2016; 48(10): 3292-8. https : //doi.org/10.1016/j.transproceed.2016.09.041

[22] Caruso R, Arrigoni C, Magon A, et al. Health determinants in italian type 2 diabetes Mellitus (T2DM) patients: A critical gender differences analysis. J Gend Stud. 2017; 7(2): 93-108. https : //doi.org/10.22381/JRGS7220176

[23] Caruso R, Rebora P, Luciani M, et al. Sex-related differences in self-care behaviors of adults with type 2 diabetes mellitus. Endocr. 2020; 67(2): 354-62. https://doi.org/10.1007/s12020-020 -02189-5

[24] Mogre V, Abanga ZO, Tzelepis F, et al. Adherence to and factors associated with self-care behaviours in type 2 diabetes patients in Ghana. BMC endocr disord. 2017; 17(20). https: //doi .org/10 $.1186 / \mathrm{s} 12902-017-0169-3$

[25] Franz MJ, MacLeod J, Evert A, et al. Academy of nutrition and dietetics nutrition practice guideline for Type 1 and Type 2 Diabetes in adults: Systematic review of evidence for medical nutrition therapy effectiveness and recommendations for integration into the nutrition care process. J Acad Nutr Diet. 2017; 117(10): 1659-79. https://doi.org/10.1016/j.jand.2017.03.022

[26] Al-Keilani MS, Almomani BA, Al-Sawalha NA, et al. Selfmonitoring of blood glucose among patients with diabetes in Jordan: Perception, adherence, and influential factors. Diabetes Res Clin Pract. 2017; 126: 79-85. https://doi.org/10.1016/j.diabre s.2017.01.005

[27] Sari Y, Upoyo AS, Isworo A, et al. Foot self-care behavior and its predictors in diabetic patients in Indonesia. BMC Res Notes. 2020; 13(38). https ://doi.org/10.1186/s13104-020-4903-y

[28] Basu S, Garg S, Sharma N, et al. Adherence to self-care practices, glycemic status and influencing factors in diabetes patients in a tertiary care hospital in Delhi. World J Diabetes. 2018; 9(5): 72-9. https://doi.org/10.4239/wjd.v9.i5.72

[29] Borba AKOT, Arruda IKG, Marques APO, et al. Knowledge and attitude about diabetes self-care of older adults in primary health care. Cien Saude Colet. 2019; 24(1). https://doi.org/10.1590/14 13-81232018241.35052016 